\title{
Innate Lymphoid Cells in Type 2 Immune Responses
}

\author{
Ananda S. Mirchandani $\cdot$ Robert J. Salmond
}

Received: 25 September 2014/ Accepted: 26 November 2014/Published online: 20 December 2014

(C) L. Hirszfeld Institute of Immunology and Experimental Therapy, Wroclaw, Poland 2014

\begin{abstract}
In recent years, several distinct innate lymphoid cell populations (ILC) have been characterized in mice and humans. Group 2 ILC function as a rapid responder population in type 2 immune responses. Thus, a wealth of data has implicated an important role for ILC2 in immunity to parasitic infection and in immune pathology in inflammatory and allergic responses. In this review, we describe recent progress in our understanding of the development and ontogeny of ILC2 populations and the mechanisms by which these cells function in a variety of infection and disease settings. Finally, we emphasize recent findings indicating functional interactions between these innate cells and their adaptive $\mathrm{CD} 4^{+} \mathrm{Th} 2$ cell counterparts.
\end{abstract}

Keywords Innate lymphoid cell $\cdot$ Th2 Parasite

Allergy

\section{Introduction}

Cytokines produced by both the innate and adaptive arms of the immune system are critical for protective immunity to infection. However, expansion of sufficient numbers of antigen-specific $\mathrm{T}$ helper (Th) cells, from rare naïve progenitors to mediate such responses typically takes 5-10 days. Therefore, innate cell types are critical for the

\footnotetext{
A. S. Mirchandani

Institute of Immunology, Infection and Inflammation, University of Glasgow, Glasgow G12 8TA, UK

\section{R. J. Salmond ( $\square)$}

Institute of Immunology and Infection Research, Ashworth Laboratories, The King's Buildings, University of Edinburgh, West Mains Road, Edinburgh EH9 3JT, UK

e-mail: robert.salmond@ed.ac.uk
}

early control of infection, particularly at mucosal and barrier surfaces. In recent years, much interest has focused on the role of several distinct groups of innate lymphoid cell (ILC) in immune responses. Similar to $\mathrm{CD} 4^{+} \mathrm{T}$ cells, these ILC populations have been classified by their expression of specific transcription factors (TFs) and effector cytokines (Spits et al. 2013). Group 1 ILC (ILC1) produce IFN- $\gamma$ and are thought to be dependent on expression of the Th1-associated TF T-bet, whilst development of IL-17/IL-22-secreting group 3 ILC (ILC3) requires retinoic acid receptor-related orphan receptor (ROR) $\gamma$ t. By contrast, group 2 ILC (ILC2) are distinguished by their expression of type 2-associated cytokines including IL-5 and IL-13. The focus of the current review is on the recent progress in our understanding of the development of ILC2 and their role in protective immunity and inflammation. For recent reviews of other aspects of ILC biology, we recommend other recent excellent articles (Hoyler et al. 2013; Romera-Hernandez et al. 2013; Sonnenberg and Artis 2012; Tait Wojno and Artis 2012; Vosshenrich and Di Santo 2013).

\section{Identification and Characterization of ILC2}

An initial description of a novel innate source of type 2 cytokines came from McKenzie, Fallon and colleagues in 2006 (Fallon et al. 2006). These researchers showed that infection of mice with the nematode parasite Nippostrongylus brasiliensis induced a non $\mathrm{B} /$ non $\mathrm{T}$ cell population that produced high levels of IL-4, IL-5 and IL13 (Fallon et al. 2006). Genetic deficiency in the IL-17 family cytokine IL-25 resulted in a failure to induce this novel population and was associated with inefficient clearance of the parasite. Subsequently, in 2010, several 
groups independently characterized populations of type 2 cytokine-producing ILC induced by epithelial-derived cytokines including IL-25 and the IL-1 family member IL33. Moro et al. (2010) described a subset of lymphoid cells that lacked expression of common lineage-defining cell surface antigens $\left(\mathrm{Lin}^{-}\right)$present in fat-associated lymphoid clusters (FALC) and named them natural helper cells. These cells expanded in response to IL-33 and were found to enhance gut helminth expulsion. Similarly, Price et al. (2010) and Neill et al. (2010) identified Lin ${ }^{-}$ILC populations that expressed IL-33 and IL-25 receptors in gut and lung tissues, using IL-4 and IL-13 reporter mice, respectively. These cells were also shown to be important in mediating gut parasite expulsion and were named innate helper cells (Price et al. 2010) and nuocytes (Neill et al. 2010) by these respective groups.

The cells described by all three papers shared numerous similarities, including expression of cell surface markers CD45, CD90, inducible co-stimulator, variable levels of cKit and Sca-1, and the cytokines receptors CD25 (IL2R $\alpha$ ), CD127 (IL-7R $\alpha$ ), IL-17RB (IL-25R), and T1/ST2 (IL-33R). These cells were further distinguished by their capacity for producing type- 2 cytokines (mainly IL-5 and IL-13) in response to IL-2, IL-25 and/or IL-33, which contributed to their ability to mediate their roles in gut parasite clearance. Importantly, an IL-25/IL-33-responsive ILC2 population was also identified in human lung and gut by Spits and colleagues (Mjosberg et al. 2011).

\section{Development and Ontogeny of ILC2}

Much recent research has focused on the developmental requirements of ILC2. These cells are found in normal or even elevated numbers in T/B cell-deficient $\left(\mathrm{Ragl}^{-/-}\right.$and $\operatorname{Rag} 2^{-/-}$) and athymic (nu/nu) mice, distinguishing their developmental requirements from those of conventional $\mathrm{T}$ and B lymphocytes (Moro et al. 2010; Neill et al. 2010). Similar to other classes of lymphocytes, ILC2 were found to originate from common lymphoid progenitor (CLP) cells (Yang et al. 2011) that develop from more immature progenitors via fms-related tyrosine kinase 3 (Flt3) signaling (Sitnicka et al. 2002). The expression of inhibitor of DNA binding 2 (Id2), which suppresses the $\mathrm{B}$ and $\mathrm{T}$ cell potential of the CLP (Ji et al. 2008), is essential for the development of ILC2 (Moro et al. 2010). Using Id2-green fluorescent protein reporter mice, Diefenbach and colleagues recently identified an $\mathrm{Id} 2^{+} \mathrm{Lin}-\mathrm{CD} 127^{+} \alpha 4 \beta 7^{+}$progenitor cell in mouse bone marrow that appears to function as a committed precursor for all ILC populations (Klose et al. 2014). Approximately $50 \%$ of these cells expressed high levels of the promyelocytic leukemia zinc finger (PLZF) protein TF that was independently identified as a marker for an ILC progenitor (ILCP) population (Constantinides et al. 2014). Two independent studies have shown a requirement for the transcription factor nuclear factor, IL-3 (Nfil3, also known as E4BP4) in the development of all ILC populations (Geiger et al. 2014; Seillet et al. 2014). Thus ILC1, ILC2 and ILC3 populations are absent or present at greatly reduced frequencies in $\mathrm{N}_{\mathrm{f}} \mathrm{3}^{-/-}$mice, a consequence of a requirement for Nfil3 expression during CLP to ILCP transition (Geiger et al. 2014). Subsequently,

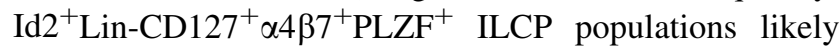
give rise to an ILC2-commited ILC2 progenitor population that expresses high levels of CD25 and T1/ST2 (Hoyler et al. 2012).

Whilst the transcriptional programs required for ILC2 development and function are not fully understood, a role for a number of TFs and cytokines has been identified. In this regard, IL-7 is required for the development of all ILC populations with the exception of conventional NK cells and recently described ILC1 populations (Klose et al. 2014). By contrast, the retinoic acid receptor-related ROR $\alpha$ has been shown to be a key TF solely for ILC2 development as in Rora mutant mice numbers of ILC2, but not other ILC populations, are markedly reduced (Halim et al. 2012; Wong et al. 2012). Additionally, CLP require IL-33 and Notch signaling to develop into ILC2 in vitro. Indeed, using a pan-Notch inhibitor, Yang et al. (2013) demonstrated that Notch signaling was important in ILC2 development in vivo. Downstream from Notch, signaling through the $\mathrm{T}$ cell factor (TCF)-1 TF was vital for ILC2 maturation, since $T c f 7$ (which encodes TCF-1) knockout mice lacked mature ILC2 and their associated immune responses (Yang et al. 2013). TCF-1 directly drove $I l 7 r$ expression, allowing ILC2 responsiveness to this cytokine. Forced TCF-1 expression in bone marrow cells in vitro, however, appears to by-pass the need for Notch signaling as the cells up-regulate many of the factors associated with ILC2 function independently of Notch signaling (Yang et al. 2013). Interestingly, data indicate that the role of TCF-1 in ILC development is not limited to ILC2 as numbers of ROR $\gamma \mathrm{t}^{+}$ILC3 are also reduced in the absence of this TF (Mielke et al. 2013; Yang et al. 2013).

The canonical Th2-associated TF GATA-3 is also a key factor for ILC2 development and function. Similar to the case for T cells, GATA-3 is required at a very early progenitor stage as in gata3-deficient mice, the development of all IL-7-dependent ILC populations is compromised (Serafini et al. 2014; Yagi et al. 2014). GATA-3 is also required for the maintenance and function of mature mouse (Hoyler et al. 2012) and human (Mjosberg et al. 2012) ILC2, but not other ILC populations, after development. Another key TF that determines ILC2 function is growth factor independence 1 (Gfil). Gfil is highly expressed in developing ILC2 progenitors (Spooner et al. 2013) 
mirroring gata3 expression. Furthermore, Gfi1 promotes expression of T1/ST2, whilst Gfi1-deficient ILC2 express low levels of T1/ST2 and are poorly responsive to IL-33, consequently producing lower levels of IL-5 and IL-13 in response to N. brasiliensis infection (Spooner et al. 2013).

\section{ILC2 Responses in Infection}

ILC2 play an important role in protective type 2 immune responses at mucosal and barrier surfaces. Perhaps the bestdescribed functions for ILC2 are in gut immunity and as a key effector population in anti-helminth immunity (Moro et al. 2010; Neill et al. 2010; Price et al. 2010). Moro et al. (2010) demonstrated that alymphoid $\mathrm{gc}^{-/} \mathrm{Rag}^{-/-}$mice had profoundly reduced goblet cell hyperplasia, mucus deposition and diminished levels of IL-13 in the peritoneal cavity in response to $N$. brasiliensis infection. Importantly, this defect could be reversed upon adoptive transfer of ILC2 but not $\mathrm{CD}^{+}{ }^{+} \mathrm{T}$ cells. In a similar manner, using $i l 1 \mathrm{rl}^{-/-}$(IL-33R-deficient) and $i l 17 b r^{-/-}$(IL-25R-deficient) mice, Neill et al. (2010) demonstrated that absence of either of these receptors alone delayed early helminth clearance, but this was eventually achieved with the expansion of ILC2 populations. However, when both the IL-33 and IL-25 receptors were knocked out, $N$. brasiliensis clearance was severely affected, suggesting partial functional redundancy between these cytokines. Parasite clearance was rescued by adoptive transfer of wild-type, but not IL-13-deficient, ILC2 into the double knockout mice underlying the critical importance of ILC2 as producers of this cytokine in infection (Neill et al. 2010).

Similar to their function in the gut, ILC2 are important in driving parasite clearance from the lung via production of cytokines including IL-5 and IL-13 (Turner et al. 2013; Wilhelm et al. 2011; Yasuda et al. 2012). Interestingly, Stockinger and colleagues demonstrated that ILC2 were also an important cellular source of IL-9 in vivo. In this regard, using an IL-9 reporter mouse strain, these investigators demonstrated that ILC2, and not $\mathrm{CD} 4^{+} \mathrm{Th} 9$ cells, were the main source of this cytokine in a model of airway inflammation (Wilhelm et al. 2011). Moreover, IL-9 was shown to act in an autocrine manner promoting ILC2 cell survival and function during the lung stage of $N$. brasiliensis infection (Turner et al. 2013).

Interestingly, some helminths appear to have evolved mechanisms of counteracting ILC2 activation. Secretory products of the nematode Heligmosomoides polygyrus have long been known to suppress protective type 2 immune responses during infection (reviewed in McSorley et al. 2013). Recent data from the Maizels laboratory have shown that $H$. polygyrus products can inhibit IL-33 release and subsequent activation of ILC2 in experimental models of allergy and inflammation (McSorley et al. 2014). It remains to be seen if this ability to dampen ILC2 activation is peculiar to $H$. polygyrus or a more general mechanism by which parasites evade immune clearance.

Demonstrations of the importance of type 2 cytokines in damage limitation and tissue repair have been widely reported (reviewed in Allen and Sutherland 2014). Consistent with these data, studies have shown that, in addition to acting as key players in immunity, ILC2 have important roles in the repair of tissue damage. The Artis laboratory reported that ILC2 numbers increase in the lungs of influenza virus-infected $\operatorname{Rag} 1^{-1-}$ mice; however, their depletion with anti-CD90 antibody did not affect viral replication (Monticelli et al. 2011). Instead, ILC2 were shown to support lung repair and its return to homeostasis following viral insult through their production of amphiregulin (Monticelli et al. 2011), a member of the epithelial growth factor family which functions as an immunomodulatory cytokine (Zaiss et al. 2013). Interestingly, amphiregulin is selectively expressed by $\mathrm{CD} 4^{+} \mathrm{Th} 2$ cells, but not Th1 or Th17 subsets, further emphasizing the similarity in function between ILC2 and their adaptive $\mathrm{CD} 4^{+}$Th counterparts (Zaiss et al. 2006).

By contrast, whilst ILC2 appear to have a benign and restorative effect following viral infection in the lung, they have also been implicated in the pathogenesis of lung fibrosis in animal models and are present in the lungs of patients suffering from idiopathic pulmonary fibrosis (Hams et al. 2014), a condition believed to be driven by deregulated repair mechanisms (Noble et al. 2012). Similarly, ILC2 have been implicated in the pathogenesis of hepatic fibrosis. In this regard, ILC2 production of IL-13 is critical for the pro-fibrotic effects of IL-33 in the liver (McHedlidze et al. 2013).

ILC2 are also present in high numbers in the dermis. Imaging studies have shown that ILC2 appear to "patrol" the skin slowly as compared with resident $\mathrm{T}$ cells (Roediger et al. 2013). Furthermore, ILC2 interact with other skin-resident cells, including dermal mast cells. The functional significance of these interactions has yet to be fully elucidated, nonetheless these studies are suggestive of a role for dermal ILC2 in immunosurveillance within this organ (Roediger et al. 2013).

\section{Role of ILC2 in the Pathogenesis of Allergic Inflammation}

In addition to their protective effects in type 2 immune responses to parasites, ILC2 have been implicated in the pathogenesis of allergic and atopic inflammatory diseases. Central to this effect of ILC2 is their ability to respond to innate cytokines such as IL-33. IL-33 has been implicated 
in the pathogenesis of asthma and intranasal administration of IL-33 induces a rapid inflammatory response in the lungs of recipient mice (reviewed in Mirchandani et al. 2012). Importantly, adoptive transfer of wild-type ILC2 into $i l 1 r 1^{-/-}$mice was sufficient to restore the characteristic lung inflammatory response induced by exogenous IL33 treatment (Salmond et al. 2012), implicating ILC2 as the primary IL-33-driven effector cell population.

A number of groups have demonstrated the role of ILC2 as key producers of type 2 cytokines in several different experimental lung inflammation models (Barlow et al. 2012, 2013; Bartemes et al. 2012; Chang et al. 2011; Kim et al. 2012; Scanlon and McKenzie 2012; Walker and McKenzie 2013). The activation of ILC2 within lungs is very rapid, with high levels of IL-5 and IL-13 production within $12 \mathrm{~h}$ of exposure of mice to the fungal allergen Alternaria alternata (Bartemes et al. 2012). Interestingly, IL-33 is a more potent trigger of the deleterious inflammatory effects of ILC2 function in the lungs as compared to IL-25 (Barlow et al. 2013). Furthermore, signals induced by IL-33 and thymic stromal lymphopoietin (TSLP) are required for the immune pathology mediated by ILC2 under conditions of chronic allergen exposure (Iijima et al. 2014). TSLP may also render lung ILC 2 resistant to corticosteroids, suggesting this pathway might represent a future therapeutic target (Kabata et al. 2013).

In addition to the role for environmental allergens in lung inflammation, respiratory tract viral infections are also potent triggers for asthma exacerbations. Chang et al. (2011) showed that ILC2, activated by macrophagederived IL-33, were sufficient to drive influenza-induced airway hyperresponsiveness (AHR) in mice. This effect was mediated by ILC2-derived IL-13, since adoptive transfer of wild-type ILC2 into infected $i l 13^{-/-}$mice was sufficient to restore viral-induced AHR in these otherwise AHR-resistant mice (Chang et al. 2011). Whilst the physiological role of ILC2 in human airway inflammation is not fully understood, ILC2 were shown to be elevated in nasal biopsies of chronic rhinosinusitis patients as compared to healthy controls (Mjosberg et al. 2011) demonstrating an association of these cells with human disease. Moreover, a recent report has shown that the prevalence of ILC2 in the blood of allergic asthma patients was substantially higher than either allergic rhinitis patients or healthy controls (Bartemes et al. 2014). These results are consistent with a role for ILC2 in the immunopathology of allergic asthma in humans.

Recent evidence has also pointed towards a role for ILC2 in type 2 inflammation in the skin. In this regard, cutaneous allergic inflammation and dermatitis can be elicited by IL-2-induced proliferation of skin-resident ILC2 (Roediger et al. 2013), or via IL-33/IL-25- and/or TSLPdriven ILC2 responses (Imai et al. 2013; Kim et al. 2012;
Salimi et al. 2013). Furthermore, human ILC2 express skin-homing receptors and are elevated in atopic lesions from human patients (Salimi et al. 2013). Together these data indicate that, like their adaptive $\mathrm{CD} 4^{+} \mathrm{Th} 2$ counterparts, ILC2 activation can be a double-edged sword eliciting both beneficial and damaging type 2 immune responses.

\section{ILC2 Interactions with Innate Cells}

In addition to modulating the activity of non-immune cells such as epithelial goblet cells, cytokine production by ILC2 has profound effects on important immune effector cell populations. One such example was identified by Koyasu and colleagues in their original description of FALCassociated "natural helper cells". These investigators identified IL-5 production by ILC2 as sufficient to induce B1 cell proliferation in vitro, an effect also seen in vivo when B1 cells were co-transferred with ILC2 into $g c^{-/-} \mathrm{Rag}^{-/-}$mice (Moro et al. 2010). Recently, the Locksley laboratory investigated the interactions of ILC2 and eosinophils using an IL-5 reporter mouse strain, in which IL-5-expressing cells could be specifically deleted. They demonstrated that, during homeostasis, lung ILC2 constitutively express IL-5; whereas IL-13 is upregulated following triggers such as parasitic infection (Nussbaum et al. 2013). Few eosinophils were present in the lung under homeostatic conditions; however, following ILC2 stimulation, upregulation of IL-13 and eotaxin-1 (CCL11) occurred, leading to eosinophil recruitment. Deletion of ILC2 in $\mathrm{Ragl}^{-1-}$ mice reduced eotaxin levels and this effect was partially restored with exogenous IL-13. Interestingly, within the intestine, where eosinophils are present at baseline, IL-5 levels followed a circadian pattern that in turn correlated with blood eosinophil numbers. The authors demonstrated that intestinal ILC2 responded to vasoactive peptides (VIP) released in response to food ingestion by producing IL-5 (Nussbaum et al. 2013). Together these data suggest that blood eosinophil circadian patterns are regulated through a VIP-IL-5 intestinal ILC2 pathway.

Further studies demonstrated that ILC2 present in visceral adipose tissue (VAT) regulate VAT eosinophil numbers through IL-5 production (Molofsky et al. 2013). VAT ILC2 activation by IL-33 or IL-25 led to increases in both eosinophil and alternatively activated macrophage (AAM) numbers (Hams et al. 2013; Molofsky et al. 2013). These studies have highlighted an important role for ILC2 in regulating obesity and glucose tolerance. Specifically, depletion of ILC2 elevated weight gain whereas treating obese mice with IL-25, thereby driving ILC2 responses, induced weight loss (Hams et al. 2013). It is possible that ILC2 production of IL-13 enhances AAM polarization in 
adipose tissue and thus serves to protect from metabolic dysregulation and obesity (Odegaard et al. 2007).

\section{ILC2 Interactions with T Cells}

Since their initial discovery ILC2 have been regarded as an innate counterpart to adaptive $\mathrm{CD} 4^{+} \mathrm{Th} 2$ cells, however, an important question was how and when do these two cell types functionally interact? In recent months several important studies have shown that ILC2 and Th2 cells reciprocally modulate the others' function and that ILC2 are important in the induction of an effective antigenspecific Th2 response. An early clue that ILC 2 and $C D 4^{+} \mathrm{T}$ cells might interact came from the finding that exogenous IL-2 potently induced ILC2 proliferation (Moro et al. 2010). We, and others, recently confirmed that antigendriven $\mathrm{CD}^{+} \mathrm{T}$ cell-derived IL-2 directly induces ILC2 proliferation and cytokine production in co-cultures (Mirchandani et al. 2014; Oliphant et al. 2014). Most interestingly, in these in vitro experiments, it was shown that ILC2 could directly affect $\mathrm{CD} 4^{+} \mathrm{T}$ cell activation via MHC II-dependent peptide presentation. Importantly ILC2 express both MHC class II molecules and co-stimulatory ligands (Oliphant et al. 2014) and are, thus, capable of providing both "signal 1" and "signal 2" required for the activation of naïve T cells. Of note, ILC2 appear to preferentially induce Th2 and oppose Th1 polarization of naïve $\mathrm{T}$ cells in a contact-dependent manner (Mirchandani et al. 2014); however, the molecular mechanisms behind this effect are yet to be fully elucidated.

ILC2 and T cell interactions were also shown to be functionally relevant in vivo. Thus, co-transfer of ILC2 markedly enhanced ovalbumin-specific $\mathrm{T}$ cell responses in the lungs of recipient mice (Mirchandani et al. 2014). Furthermore, depletion of ILC2 diminishes adaptive Th2 responses to inhaled allergens (Gold et al. 2014; Halim et al. 2014) and parasites (Oliphant et al. 2014). It is likely that ILC2 promote $\mathrm{CD}^{+}{ }^{+} \mathrm{Th} 2$ responses by both cytokinedependent and -independent processes. In this regard, following intranasal papain delivery, ILC2-derived IL-13 indirectly enhances $\mathrm{T}$ cell priming by promoting dendritic cell migration to draining lymph nodes (Halim et al. 2014). By contrast MHC class II-deficient ILC2 are incapable of restoring Th2 responses in N. brasiliensis-infected ILC2depleted mice, indicating an important role for direct Agpresentation by ILC2 in vivo (Oliphant et al. 2014).

\section{Conclusions and Future Prospects}

The study of ILC2 and other ILC populations has progressed at a dramatic pace in the last half decade. Since their initial characterization in 2010, important roles for ILC2 in numerous infectious and disease settings have been reported. A prior impediment to studying ILC2 function in vivo was the lack of animal models specifically lacking this cell type. However, recently described mouse strains allowing inducible deletion of ILC2 should allow the physiological roles of these populations in vivo to be fully dissected (Nussbaum et al. 2013; Oliphant et al. 2014). Further important questions concern the function of ILC2 in human immunity and disease. Do they fulfill the same roles as described in animal studies and can their function be therapeutically manipulated? It is anticipated that the study of these cell populations will continue to provide insight into type 2 immune responses in the coming years.

Acknowledgments A.S.M. was supported by a Medical Research Council UK Clinical Training Fellowship.

\section{References}

Allen JE, Sutherland TE (2014) Host protective roles of type 2 immunity: parasite killing and tissue repair, flip sides of the same coin. Semin Immunol 26:329-340

Barlow JL, Bellosi A, Hardman CS et al (2012) Innate IL-13producing nuocytes arise during allergic lung inflammation and contribute to airways hyperreactivity. J Allergy Clin Immunol 129(191-198):e1-e4

Barlow JL, Peel S, Fox J et al (2013) IL-33 is more potent than IL-25 in provoking IL-13-producing nuocytes (type 2 innate lymphoid cells) and airway contraction. J Allergy Clin Immunol 132:933-941

Bartemes KR, Iijima K, Kobayashi T et al (2012) IL-33-responsive lineage- CD25+ CD44(hi) lymphoid cells mediate innate type 2 immunity and allergic inflammation in the lungs. J Immunol 188:1503-1513

Bartemes KR, Kephart GM, Fox SJ et al (2014) Enhanced innate type 2 immune response in peripheral blood from patients with asthma. J Allergy Clin Immunol 134(671-678):e4

Chang YJ, Kim HY, Albacker LA et al (2011) Innate lymphoid cells mediate influenza-induced airway hyper-reactivity independently of adaptive immunity. Nat Immunol 12:631-638

Constantinides MG, McDonald BD, Verhoef PA et al (2014) A committed precursor to innate lymphoid cells. Nature 508:397-401

Fallon PG, Ballantyne SJ, Mangan NE et al (2006) Identification of an interleukin (IL)-25-dependent cell population that provides IL-4, IL-5, and IL-13 at the onset of helminth expulsion. J Exp Med 203:1105-1116

Geiger TL, Abt MC, Gasteiger G et al (2014) Nfil3 is crucial for development of innate lymphoid cells and host protection against intestinal pathogens. J Exp Med 211:1723-1731

Gold MJ, Antignano F, Halim TY et al (2014) Group 2 innate lymphoid cells facilitate sensitization to local, but not systemic, TH2-inducing allergen exposures. J Allergy Clin Immunol 133:1142-1148

Halim TY, MacLaren A, Romanish MT et al (2012) Retinoic-acidreceptor-related orphan nuclear receptor alpha is required for natural helper cell development and allergic inflammation. Immunity 37:463-474 
Halim TY, Steer CA, Mathä L et al (2014) Group 2 innate lymphoid cells are critical for the initiation of adaptive $\mathrm{T}$ helper 2 cellmediated allergic lung inflammation. Immunity 40:425-435

Hams E, Locksley RM, McKenzie AN et al (2013) Cutting edge: IL25 elicits innate lymphoid type 2 and type II NKT cells that regulate obesity in mice. J Immunol 191:5349-5353

Hams E, Armstrong ME, Barlow JL et al (2014) IL-25 and type 2 innate lymphoid cells induce pulmonary fibrosis. Proc Natl Acad Sci USA 111:367-372

Hoyler T, Klose CS, Souabni A et al (2012) The transcription factor GATA-3 controls cell fate and maintenance of type 2 innate lymphoid cells. Immunity 37:634-648

Hoyler T, Connor CA, Kiss EA et al (2013) T-bet and Gata3 in controlling type 1 and type 2 immunity mediated by innate lymphoid cells. Curr Opin Immunol 25:139-147

Iijima K, Kobayashi T, Hara K et al (2014) IL-33 and thymic stromal lymphopoietin mediate immune pathology in response to chronic airborne allergen exposure. J Immunol 193:1549-1559

Imai Y et al (2013) Skin-specific expression of IL-33 activates group 2 innate lymphoid cells and elicits atopic dermatitis-like inflammation in mice. Proc Natl Acad Sci USA 110:13921-13926

Ji M, Li H, Suh HC et al (2008) Id2 intrinsically regulates lymphoid and erythroid development via interaction with different target proteins. Blood 112:1068-1077

Kabata H, Moro K, Fukunaga K et al (2013) Thymic stromal lymphopoietin induces corticosteroid resistance in natural helper cells during airway inflammation. Nat Commun 4:2675

Kim HY, Chang YJ, Subramanian S et al (2012) Innate lymphoid cells responding to IL-33 mediate airway hyperreactivity independently of adaptive immunity. J Allergy Clin Immunol 129(216-227):e1-e6

Klose CS, Flach M, Möhle L et al (2014) Differentiation of type 1 ILCs from a common progenitor to all helper-like innate lymphoid cell lineages. Cell 157:340-356

McHedlidze T, Waldner M, Zopf S et al (2013) Interleukin-33dependent innate lymphoid cells mediate hepatic fibrosis. Immunity 39:357-371

McSorley HJ, Hewitson JP, Maizels RM (2013) Immunomodulation by helminth parasites: defining mechanisms and mediators. Int $\mathbf{J}$ Parasitol 43:301-310

McSorley HJ, Blair NF, Smith KA et al (2014) Blockade of IL-33 release and suppression of type 2 innate lymphoid cell responses by helminth secreted products in airway allergy. Mucosal Immunol 7:1068-1078

Mielke LA, Groom JR, Rankin LC et al (2013) TCF-1 controls ILC2 and NKp46+RORgammat+ innate lymphocyte differentiation and protection in intestinal inflammation. $\mathrm{J}$ Immunol 191:4383-4391

Mirchandani AS, Salmond RJ, Liew FY (2012) Interleukin-33 and the function of innate lymphoid cells. Trends Immunol 33:389-396

Mirchandani AS, Besnard AG, Yip E et al (2014) Type 2 innate lymphoid cells drive CD4 + Th2 cell responses. J Immunol 192:2442-2448

Mjosberg JM, Trifari S, Crellin NK et al (2011) Human IL-25- and IL-33-responsive type 2 innate lymphoid cells are defined by expression of CRTH2 and CD161. Nat Immunol 12:1055-1062

Mjosberg J, Bernink J, Golebski K et al (2012) The transcription factor GATA3 is essential for the function of human type 2 innate lymphoid cells. Immunity 37:649-659

Molofsky AB, Nussbaum JC, Liang HE et al (2013) Innate lymphoid type 2 cells sustain visceral adipose tissue eosinophils and alternatively activated macrophages. J Exp Med 210:535-549

Monticelli LA, Sonnenberg GF, Abt MC et al (2011) Innate lymphoid cells promote lung-tissue homeostasis after infection with influenza virus. Nat Immunol 12:1045-1054
Moro K, Yamada T, Tanabe M et al (2010) Innate production of $\mathrm{T}(\mathrm{H}) 2$ cytokines by adipose tissue-associated c-Kit $(+) \mathrm{Sca}-1(+)$ lymphoid cells. Nature 463:540-544

Neill DR, Wong SH, Bellosi A et al (2010) Nuocytes represent a new innate effector leukocyte that mediates type- 2 immunity. Nature 464:1367-1370

Noble PW, Barkauskas CE, Jiang D (2012) Pulmonary fibrosis: patterns and perpetrators. J Clin Invest 122:2756-2762

Nussbaum JC, Van Dyken SJ, von Moltke J et al (2013) Type 2 innate lymphoid cells control eosinophil homeostasis. Nature 502:245-248

Odegaard JI, Ricardo-Gonzalez RR, Goforth MH et al (2007) Macrophage-specific PPARgamma controls alternative activation and improves insulin resistance. Nature 447:1116-1120

Oliphant CJ, Hwang YY, Walker JA et al (2014) MHCII-mediated dialog between group 2 innate lymphoid cells and CD4(+) T cells potentiates type 2 immunity and promotes parasitic helminth expulsion. Immunity 41:283-295

Price AE, Liang HE, Sullivan BM et al (2010) Systemically dispersed innate IL-13-expressing cells in type 2 immunity. Proc Natl Acad Sci USA 107:11489-11494

Roediger B, Kyle R, Yip KH et al (2013) Cutaneous immunosurveillance and regulation of inflammation by group 2 innate lymphoid cells. Nat Immunol 14:564-573

Romera-Hernandez M, Aparicio-Domingo P et al (2013) Damage control: rorgammat + innate lymphoid cells in tissue regeneration. Curr Opin Immunol 25:156-160

Salimi M, Barlow JL, Saunders SP et al (2013) A role for IL-25 and IL-33-driven type-2 innate lymphoid cells in atopic dermatitis. J Exp Med 210:2939-2950

Salmond RJ, Mirchandani AS, Besnard AG et al (2012) IL-33 induces innate lymphoid cell-mediated airway inflammation by activating mammalian target of rapamycin. J Allergy Clin Immunol 130(1159-1166):e6

Scanlon ST, McKenzie AN (2012) Type 2 innate lymphoid cells: new players in asthma and allergy. Curr Opin Immunol 24:707-712

Seillet C, Rankin LC, Groom JR et al (2014) Nfil3 is required for the development of all innate lymphoid cell subsets. J Exp Med 211:1733-1740

Serafini N, Klein Wolterink RG et al (2014) Gata3 drives development of RORgammat + group 3 innate lymphoid cells. J Exp Med 211:199-208

Sitnicka E, Bryder D, Theilgaard-Monch K et al (2002) Key role of flt3 ligand in regulation of the common lymphoid progenitor but not in maintenance of the hematopoietic stem cell pool. Immunity 17:463-472

Sonnenberg GF, Artis D (2012) Innate lymphoid cell interactions with microbiota: implications for intestinal health and disease. Immunity 37:601-610

Spits H, Artis D, Colonna M et al (2013) Innate lymphoid cells-a proposal for uniform nomenclature. Nat Rev Immunol 13:145-149

Spooner CJ, Lesch J, Yan D et al (2013) Specification of type 2 innate lymphocytes by the transcriptional determinant Gfil. Nat Immunol 14:1229-1236

Tait Wojno ED, Artis D (2012) Innate lymphoid cells: balancing immunity, inflammation, and tissue repair in the intestine. Cell Host Microbe 12:445-457

Turner JE, Morrison PJ, Wilhelm C et al (2013) IL-9-mediated survival of type 2 innate lymphoid cells promotes damage control in helminth-induced lung inflammation. J Exp Med 210:2951-2965

Vosshenrich CA, Di Santo JP (2013) Developmental programming of natural killer and innate lymphoid cells. Curr Opin Immunol 25:130-138 
Walker JA, McKenzie AN (2013) Development and function of group 2 innate lymphoid cells. Curr Opin Immunol 25:148-155

Wilhelm C, Hirota K, Stieglitz B et al (2011) An IL-9 fate reporter demonstrates the induction of an innate IL-9 response in lung inflammation. Nat Immunol 12:1071-1077

Wong SH, Walker JA, Jolin HE et al (2012) Transcription factor RORalpha is critical for nuocyte development. Nat Immunol 13:229-236

Yagi R, Zhong C, Northrup DL et al (2014) The transcription factor GATA3 is critical for the development of all IL-7Ralphaexpressing innate lymphoid cells. Immunity 40:378-388

Yang Q, Saenz SA, Zlotoff DA et al (2011) Cutting edge: natural helper cells derive from lymphoid progenitors. J Immunol 187:5505-5509
Yang Q, Monticelli LA, Saenz SA et al (2013) T cell factor 1 is required for group 2 innate lymphoid cell generation. Immunity 38:694-704

Yasuda K, Muto T, Kawagoe T et al (2012) Contribution of IL-33activated type II innate lymphoid cells to pulmonary eosinophilia in intestinal nematode-infected mice. Proc Natl Acad Sci USA 109:3451-3456

Zaiss DM, Yang L, Shah PR et al (2006) Amphiregulin, a TH2 cytokine enhancing resistance to nematodes. Science 314:1746

Zaiss DM, van Loosdregt J, Gorlani A et al (2013) Amphiregulin enhances regulatory $\mathrm{T}$ cell-suppressive function via the epidermal growth factor receptor. Immunity 38:275-284 